\title{
Lactato mínimo em protocolo de rampa e sua validade em estimar o máximo estado estável de lactato
}

\author{
Lactate minimum in a ramp protocol and its validity to estimate \\ the maximal lactate steady state
}

\author{
Emerson Pardono ${ }^{1}$ \\ Bibiano Madrid \\ Daisy Fonseca Motta 1 \\ Márcio Rabelo Mota ${ }^{1}$ \\ Carmen Silvia Grubert Campbell ${ }^{1}$ \\ Herbert Gustavo Simões 1
}

1 Universidade Católica de Brasília. Programa de Pós-Graduação em Educação Física. Brasília, DF. Brasil.

Recebido em 06/02/08 Revisado em 17/07/08 Aprovado em 12/11/08
Resumo - Os objetivos foram verificar a validade do lactato mínimo (LM) modelo rampa em determinar a intensidade de LM (ILM), bem como estimar uma intensidade de exercício correspondente ao máximo estado estável de lactato (IMEEL). Ainda, a possibilidade de identificar parâmetros de aptidão aeróbia e anaeróbia em um único teste de LM em ciclistas recreacionais. Participaram do estudo 14 ciclistas de nível regional, do sexo masculino, submetidos ao protocolo de LM em cicloergômetro (Excalibur-Lode), composto por um teste incremental com carga inicial de 75 watts e incrementos de 1 Watt a cada 6 segundos. A indução prévia de hiperlactatemia foi realizada através do teste anaeróbio de Wingate (TAW) (Monark-834E), com carga de 8,57\% da massa corporal. Foram obtidos a potência pico $(11,5 \pm 2,0 \mathrm{Watts} / \mathrm{kg})$, potência média $(9,8 \pm 1,7 \mathrm{Watts} / \mathrm{kg})$, índice de fadiga $(33,7 \pm 7 \%)$ e lactatemia $7 \mathrm{~min}$ após o TAW $\left(10,5 \pm 2,3 \mathrm{mmol} . \mathrm{L}^{-1}\right)$. O teste incremental identificou a ILM (209,1 $\pm 23,2$ Watts), e respectivas concentração de lactato $\left(2,9 \pm 0,7 \mathrm{mmol} . \mathrm{L}^{-1}\right)$, frequência cardíaca $(153,6 \pm 10,6 \mathrm{bpm})$, e também a potência máxima (305,2 \pm 31 Watts). A IMEEL foi determinada a partir de 2 a 4 séries de 30 min em intensidade constante (207,8 $\pm 17,7$ Watts), não diferindo da ILM, apresentando boa concordância entre si. $\mathrm{O}$ teste LM em protocolo de rampa parece valido em identificar a ILM e estimar a intensidade do MEEL de ciclistas regionais. Ainda, parâmetros de aptidão anaeróbia e aeróbia também foram identificados durante uma única sessão.

Palavras-chave: Avaliação; Aptidão física; Lactato.

Abstract - The objectives of this study were to evaluate the validity of the lactate minimum (LM) using a ramp protocol for the determination of LM intensity (LMI), and to estimate the exercise intensity corresponding to maximal blood lactate steady state (MLSS). In addition, the possibility of determining aerobic and anaerobic fitness was investigated. Fourteen male cyclists of regional level performed one LM protocol on a cycle ergometer (Excalibur-Lode) consisting of an incremental test at an initial workload of 75 Watts, with increments of 1 Watt every 6 seconds. Hyperlactatemia was induced by a 30-second Wingate anaerobic test (WAT) (Monark-834E) at a workload corresponding to $8.57 \%$ of the volunteer's body weight. Peak power (11.5 \pm 2 Watts/ $\mathrm{kg})$, mean power output $(9.8 \pm 1.7 \mathrm{Watts} / \mathrm{kg})$, fatigue index $(33.7 \pm 2.3 \%)$ and lactate 7 min after WAT $(10.5 \pm 2.3 \mathrm{mmol} / \mathrm{L})$ were determined. The incremental test identified LMI $(207.8 \pm 17.7$ Watts) and its respective blood lactate concentration $(2.9 \pm 0.7 \mathrm{mmol} / \mathrm{L})$, heart rate $(153.6 \pm 10.6$ bpm), and also maximal aerobic power (305.2 \pm 31.0 Watts). MLSS intensity was identified by 2 to 4 constant exercise tests $(207.8 \pm 17.7$ Watts), with no difference compared to LMI and good agreement between the two parameters. The LM test using a ramp protocol seems to be a valid method for the identification of LMI and estimation of MLSS intensity in regional cyclists. In addition, both anaerobic and aerobic fitness parameters were identified during a single session. Key words: Evaluation; Aerobic fitness; Lactate. 


\section{INTRODUÇÃO}

Parâmetros de aptidão aeróbia e anaeróbia apresentam relação com o desempenho no ciclismo ${ }^{1,2}$, e são importantes para a prescrição de treinamento. A aptidão anaeróbia pode ser obtida a partir de protocolos específicos como o teste anaeróbio de Wingate (TAW) ${ }^{3}$, enquanto a aptidão aeróbia pode ser determinada pelo interessante protocolo do lactato mínimo (LM)².

O TAW fornece indicativos do desempenho motor em situações metabólicas de predominância anaeróbia, como a potência anaeróbia máxima ou potência-pico (PP), capacidade anaeróbia ou potência-média (PM) e índice de fadiga (IF). Possui uma fácil aplicabilidade e validade reconhecida, sendo empregável em diferentes populações ${ }^{3,4}$.

O LM identifica uma intensidade de exercício sustentável, na qual se observa um equilíbrio entre produção e remoção de lactato sanguíneo ([lac] $)^{2,5,6}$. Este protocolo pode ser aplicado no ciclismo, onde a validade da intensidade de lactato mínimo (ILM) em identificar o máximo estado estável de lactato (MEEL) tem sido documentada ${ }^{5}$ durante testes com estágios incrementais escalonados ${ }^{7,8}$. Porém, a ILM em testes com incrementos do tipo rampa ${ }^{9,10}$ e sua validade em estimar o MEEL foram pouco estudadas, uma vez que a cinética de [lac] parece ser lenta quando comparada às variáveis ventilatórias. Testes incrementais escalonados, com duração dos estágios de aproximadamente 3 minutos têm sido utilizados para que haja estabilidade lactacidêmica ${ }^{5}$, o que nos remete à ideia de que testes em rampa não sejam adequados para identificar a ILM.

A cinética do [lac] durante a parte incremental do LM se diferencia dos demais testes incrementais em função da indução prévia originada por um esforço anaeróbio máximo. Alguns estudos ${ }^{7,11}$, adotaram o TAW para elevação prévia da [lac] em testes de LM, porém não investigaram as variáveis anaeróbias obtidas. Portanto, parece possível identificar a aptidão anaeróbia e aeróbia em uma única sessão experimental, minimizando visitas laboratoriais, reduzindo custos e agilizando a avaliação e prescrição de treinamento para ciclistas.

Assim, os objetivos do presente estudo foram verificar a validade do LM modelo rampa em determinar a intensidade de LM e estimar uma intensidade de exercício correspondente ao MEEL. Ainda, a possibilidade de identificar parâmetros de aptidão aeróbia e anaeróbia em um único teste de LM em ciclistas recreacionais foi investigada.

\section{PROCEDIMENTOS METODOLÓGICOS}

O presente estudo foi aprovado pelo Comitê de Ética em Pesquisa da Universidade Católica de Brasília (UCB) (CEP/UCB 011/2003). Os voluntários foram informados sobre os riscos e benefícios da participação no estudo e assinaram um termo de consentimento livre e esclarecido. Os testes foram realizados no Laboratório de Avaliação Física e Treinamento (LAFIT-UCB).

Participaram do estudo 14 ciclistas recreacionais do sexo masculino (27 $\pm 6,1$ anos; $71,1 \pm 9,8 \mathrm{~kg}$; $176,2 \pm 5,4 \mathrm{~cm} ; 12 \pm 5,2 \%$ gordura; $2,4 \pm 1,9$ anos de treinamento), orientados a não praticar exercícios físicos nas 48 horas antecedentes aos testes, não ingerirem bebida alcoólica ou com cafeína nas 24 horas anteriores e a manterem os mesmos hábitos alimentares durante todo estudo. Os testes foram realizados com intervalos de 48 horas e sempre no mesmo horário do dia. As sessões eram iniciadas após $5 \mathrm{~min}$ de alongamento e $5 \mathrm{~min}$ de aquecimento (carga de 75Watts).

\section{LM: TAW e Teste Incremental (TI):}

O protocolo de LM foi composto por duas partes. Inicialmente, foram submetidos ao TAW, onde o ciclista iniciava o teste de maneira lançada, sendo orientado a pedalar na máxima velocidade durante 30 seg contra uma resistência previamente estabelecida $(0,0857$ vezes a massa corporal), para uma potência mecânica supramáxima e indução de fadiga $^{3}$, com consequente hiperlactatemia ${ }^{7,1}$. O TAW foi realizado em um cicloergômetro com frenagem mecânica (Monark-SE, Ergomedic-834E).

O teste foi filmado $(60 \mathrm{~Hz})$, com obtenção das imagens a cada 5 segundos para a contagem do número parcial $(5 \mathrm{seg})$ e total (30seg) das repetições ${ }^{12}$. Permitindo identificar a PP, maior potência produzida. A PM é a média de todas as potências e o IF indica o decréscimo na geração de potência em relação à PP. Os coeficientes de correlação intraclasse para estas variáveis possuíram excelente reprodutibilidade (variando entre 0,79 e 0,98) de acordo com dados ainda não publicados.

A realização do TAW possibilitou, após 7min de recuperação, elevação da lactatemia para inicio do TI. Esta segunda parte do LM foi realizada em cicloergômetro (Excalibur-Lode) acionado por controle computadorizado programável. O TI começou no $8^{\circ}$ minuto de recuperação, com carga inicial de 75 watts e incrementos de 1Watt/6seg (modelo rampa), evidenciando valores de 30 Watts a cada três minutos (normalmente aplicado em incrementos 
escalonados). A frequência de pedalada permaneceu entre 60 e $70 \mathrm{rpm}$ até que o participante atingisse a exaustão voluntária máxima. O estágio em que se observou a menor [Lac] durante o TI foi considerada ILM, representando a capacidade aeróbia $(\mathrm{CA})^{11,13}$. Em alguns casos, a [Lac] obteve pouca variação entre os estágios próximos a ILM, desta maneira a função polinomial de segunda ordem foi aplicada para facilitar a identificação ${ }^{14}$. A intensidade atingida no momento da exaustão foi considerada a potência máxima (Pmax).

\section{MEEL}

Treze dos 14 participantes foram posteriormente submetidos a no mínimo 2 e no máximo 4 testes retangulares de $30 \mathrm{~min}$ em cicloergômetro (Excalibur-Lode). O primeiro teste foi randomizado, utilizando-se a ILM ou $6 \%$ acima desta, com incrementos ou reduções de $6 \%$ nas intensidades para os demais testes ${ }^{15}$. A intensidade do MEEL (IMEEL) foi determinada considerando a maior intensidade na qual a lactatemia não aumentou mais que $0,05 \mathrm{mmol} \cdot \mathrm{L}^{-1} \cdot \mathrm{min}^{-1}$ entre o $10^{\circ}$ e $30^{\circ}$ minutos de exercício ${ }^{16}$.

\section{Critérios de Coletas e Análises Sanguíneas}

As amostras sanguíneas foram coletadas no $7^{\circ} \mathrm{mi}$ nuto após o término do TAW ([Lac]-7’), bem como a cada 1,5 min para 5 voluntários e a cada 3 min para os demais voluntários durante o TI ${ }^{7}$. Padronizaramse coletas a cada $3 \mathrm{~min}$ devido à lenta cinética de [lac] observada quando as amostras foram obtidas a cada $1,5 \mathrm{~min}$, apresentando valores com variação de até $0,3 \mathrm{mmol} . \mathrm{L}^{-1}$ nas intensidades próximas ao LM (durante 2-4 estágios).

As amostras foram obtidas a partir de uma punção do lobo da orelha utilizando capilares de vidros calibrados para $25 \mu \mathrm{L}$ de sangue e depositados em micro-tubos Eppendorff contendo $50 \mu \mathrm{L}$ de fluoreto de sódio (NAF1\%) para posterior dosagem (YSI 2700-SELECT). Este equipamento eletroenzimático foi programado para realizar calibrações automáticas no início e a cada 10 dosagens, utilizando-se ainda como fator de correção uma [Lac] conhecida $(5,62 \mathrm{mmol})$.

\section{Análise Estatística}

Foi empregada análise descritiva como média (Méd) \pm desvio padrão (DP), bem como valores mínimo e máximo. Os escores das variáveis apresentaram distribuição normal, tanto para o LM (TAW e TI) $(\mathrm{n}=14)$ quanto para MEEL $(\mathrm{n}=13)$. Assim, o teste $\mathrm{t}$ de Student para dados pareados foi aplicado entre
ILM e IMEEL, assim como a técnica de concordância de Bland-Altman. O coeficiente de correlação de Pearson também foi aplicado $(\mathrm{p} \leq 0,05)$.

\section{RESULTADOS}

A [Lac] durante a realização do protocolo LM, após 7 minutos do TAW e durante o TI, bem como durante os testes de MEEL, estão representados nas figuras 1 e 2 , respectivamente.

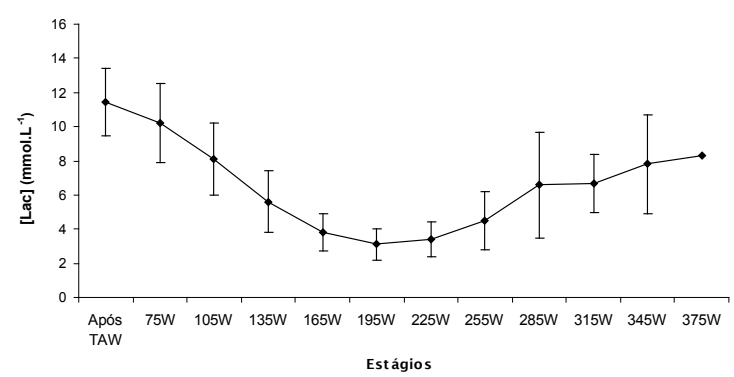

Figura 1. [Lac] durante todo o protocolo de LM. No primeiro estágio do $\mathrm{TI}$ os voluntários já estavam com hiperlactatemia

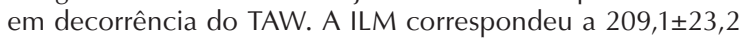
Watts $(n=14)$.

Embora o TI tenha sido rampado (1Watt/6seg), a obtenção das variáveis aconteceu em frações de $1,5$ minuto ( $n=5)$ e 3 minutos ( $n=9$ ), ou seja, a cada 15 e 30 Watts, respectivamente. Já para os testes de MEEL, as coletas foram realizadas a cada 5 minutos de exercício, onde nenhum voluntário interrompeu o teste por exaustão voluntária ou qualquer outra razão antes dos 30 minutos. A variação média de [Lac] entre o $10^{\circ}$ e $30^{\circ}$ minutos na IMEEL foi de 0,5 mmol. $L^{-1}$, enquanto esta variação na intensidade de $6 \%$ acima da IMEEL foi de 1,7 mmol..-1 (Figura 2).

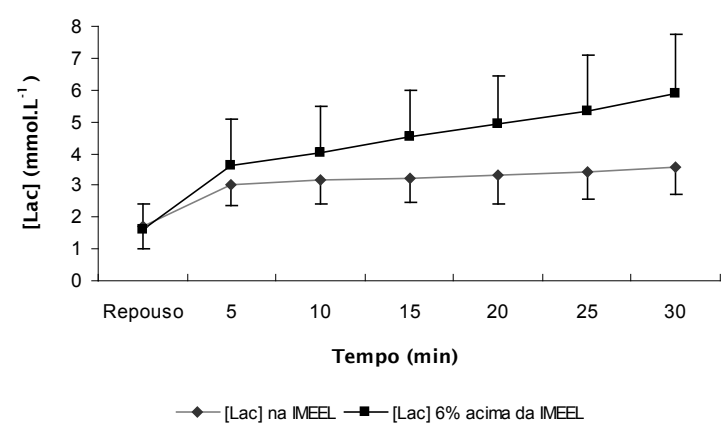

Figura 2. [Lac] durante os testes de 30 minutos na intensidade do MEEL e na intensidade de $6 \%$ acima da IMEEL. Os voluntários finalizaram o teste com intensidade de $6 \%$ acima do MEEL apresentando variação de lactatemia superior a $0,05 \mathrm{mmol} \cdot \mathrm{L}^{-1}$. $\min ^{-1}$ nos 20 minutos finais de exercício $(n=13)$.

Os valores de aptidão anaeróbia e aeróbia estão apresentados na tabela 1 . Os valores de [Lac]-7' não podem ser considerados como pico de [Lac], uma 
Tabela 1. Análise descritiva das variáveis obtidas no teste de LM ( $n=14)$ e no teste em que se obteve a IMEEL ( $n=13$ ).

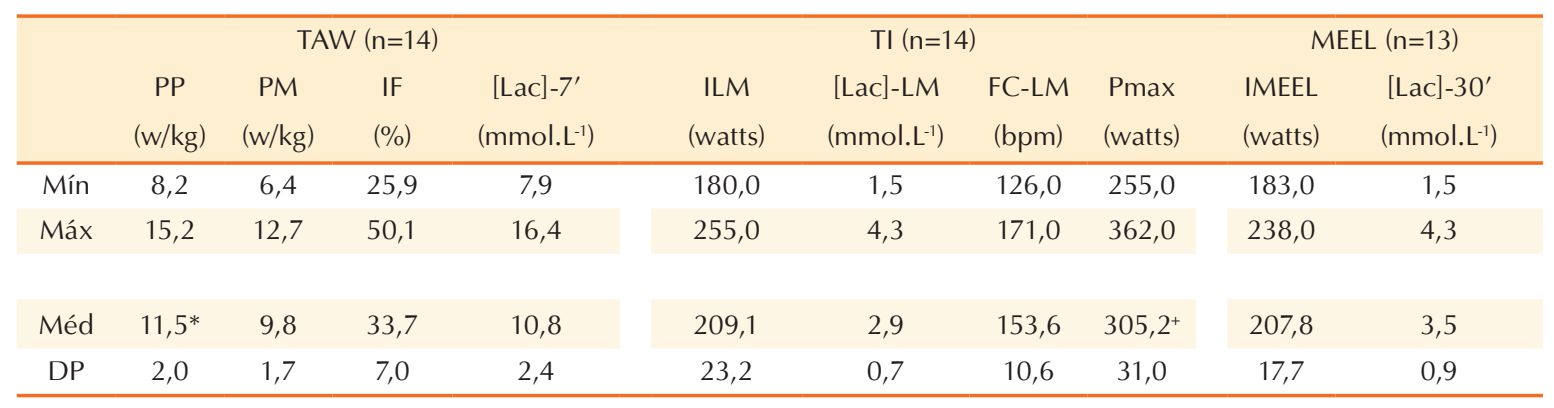

${ }^{*} \mathrm{p}<0,05$ em relação a PM; ${ }^{+} \mathrm{p}<0,05$ em relação a ILM e IMEEL.

Tabela 2. Correlação entre Pmax e IMEEL com demais variáveis obtidas pelo LM.

\begin{tabular}{|c|c|c|c|c|c|c|c|c|c|}
\hline & PP & PM & IF & [Lac]-7' & ILM & [Lac]-LM & FC-LM & [Lac]-30' & Pmax \\
\hline Pmax & $-0,49$ & $-0,46$ & $-0,21$ & $-0,35$ & $0,90^{* *}$ & $-0,45$ & $-0,54^{*}$ & $-0,59^{*}$ & ---- \\
\hline IMEEL & $-0,37$ & $-0,38$ & $-0,09$ & $-0,16$ & $0,78^{* *}$ & $-0,29$ & $-0,50$ & $-0,79 *$ & $0,66^{*}$ \\
\hline
\end{tabular}

${ }^{*} \mathrm{p}<0,05 ; * * \mathrm{p}<0,01$.

vez que não foram realizadas coletas a cada minuto para identificar este pico. As demais variáveis obtidas pelo TAW, bem como referentes a ILM e respectivos valores de lactatemia ([Lac]-LM), frequência cardíaca (FC-LM) e Pmax também estão reportadas na tabela 1 . Valores de IMEEL e [Lac] no $30^{\circ}$ minuto de teste ([Lac]-30') não diferiram da $\operatorname{ILM}(\mathrm{p}=0,83)$ e $[\operatorname{Lac}]-\operatorname{LM}(\mathrm{p}=0,10)$ respectivamente, não apresentando correlação entre esta última e a [Lac]-30' $(r=0,15)$. No entanto, a Pmax diferiu das intensidades de LM e MEEL $(\mathrm{p}<0,05)$, sendo também encontrada diferença significativa entre as variáveis anaeróbias PP e PM.

A tabela 2 apresenta as correlações existentes entre Pmax e IMEEL com as demais variáveis $(n=13)$. A Pmax se correlacionou com a ILM $(r=0,90, p<0,01), F C-L M(r=-0,54, p<0,05)$ e [Lac]$30^{\prime}(r=-0,59, p<0,05)$, enquanto a ILM, [Lac]-30' e Pmax se correlacionaram com a IMEEL $(\mathrm{r}=0,78$, $\mathrm{p}<0,01 ; \mathrm{r}=-0,79, \mathrm{p}<0,05 ; \mathrm{r}=0,66, \mathrm{p}<0,05$; respectivamente). Embora não significativa, a Pmax e IMEEL obtiveram correlações inversas com as variáveis determinadas pelo TAW.

Além das IMEEL (207,8 $\pm 17,7$ Watts) e ILM (206,9 $\pm 22,6$ Watts) não terem diferido $(\mathrm{p}=0,83)$ e obtido boa correlação $(r=0,78$; $p<0,05)$, apresentaram também boa concordância entre si pela técnica de Bland-Altman (figura 3), onde a ILM correspondeu em média a 99,3\% da IMEEL. Os resultados demonstraram que 5 voluntários $(38,0 \%$ da amostra) obtiveram as mesmas intensidades de CA em ambos os protocolos, contudo, para 4 participantes $(31,0 \%)$ a ILM apresentou valores inferiores (cerca de 8,1\%) àquelas de IMEEL, e para os outros quatro participantes $(31,0 \%)$ a ILM foi identificada acima (aproximadamente 6,0\%) da IMEEL.

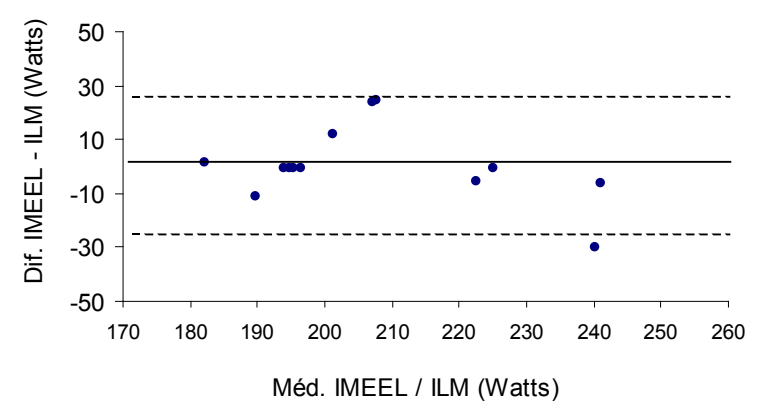

Figura 3. Análise de concordância de Bland-Altman, a linha contínua indica a média das diferenças e as linhas tracejadas representam $95 \%$ do limite de confiança entre as duas variáveis $(n=13)$.

Os resultados obtidos permitem analisar o percentual em que foram observados os valores de ILM, FC-LM, IMEEL e FC do $30^{\circ}$ minuto da IMEEL em relação à Pmax, onde a ILM e a FCLM foram observadas, em média, a 68,8\% e 85,0\% respectivamente, e os valores de IMEEL e FC do $30^{\circ}$ minuto foram observados a $69,1 \%$ e $89,0 \%$, respectivamente, da Pmax.

\section{DISCUSSÃO}

O presente estudo verificou a validade do LM modelo rampa em determinar a ILM e estimar uma intensidade de exercício correspondente ao MEEL. Ainda, a possibilidade de identificar parâmetros de aptidão aeróbia e anaeróbia em um único teste de LM em ciclistas recreacionais foi investigada. Os principais resultados demonstraram que o LM 
rampa, realizado após hiperlactatemia induzida a partir do TAW, permitiu a identificação de variáveis aeróbias e anaeróbias em uma única sessão de teste (tabela 1), onde a ILM não diferiu da IMEEL (padrão ouro), apresentando boa correlação ( $\mathrm{r}=0,78, \mathrm{p}<0,01)$ (tabela 2) e concordância entre si (figura 3).

O TAW possibilitou, além da indução da hiperlactatemia, obter variáveis como PP, PM, IF e [Lac]-7', que são importantes parâmetros da avaliação anaeróbia e que se correlacionam com o desempenho em provas de velocidade em corrida de até 200 metros $^{12}$. Os valores de [Lac]-7' em estudos que utilizaram o TAW para indução da hiperlactatemia, para a realização de teste incremental, encontraram concentrações médias ligeiramente superiores ao presente estudo (aproximadamente 12 mmol.L $\left.{ }^{-1}\right)$ em indivíduos fisicamente ativos ${ }^{11,17}$.

Quando analisados os valores de PP e PM (tabela 1), verificou-se cargas inferiores aos achados em ciclistas competidores da Federação Americana de Ciclismo (variando de 12,8 a 13,9 W/kg, 10,4 a $11,2 \mathrm{~W} / \mathrm{kg}$, respectivamente) ${ }^{18}$. Contudo, a carga utilizada no presente estudo, durante o TAW, provavelmente não foi a adequada para a amostra estudada ${ }^{3}$ o que empobrece as comparações com a literatura. Normalmente sugere-se que estas devem ficar restritas aos estudos com mesmos protocolos ${ }^{4,19}$, visto que quanto maior a carga para a realização do TAW de membros inferiores, maior a potência gerada ${ }^{19}$, interferindo nos valores de aptidão anaeróbia.

Todavia, mesmo com as limitações verificadas quanto à carga do TAW, aplicando-se valores inferiores aos sugeridos para ciclistas, importantes variáveis puderam ser discutidas, como a especificidade dentro da modalidade, tipo de treinamento e possíveis características das células musculares. Os ciclistas eram predominantemente passistas (78,5\%), sendo submetidos a treinamentos de maior volumes em relação à intensidade, semelhante às características dos treinos realizados por corredores fundistas ${ }^{20}$. Além disso, devido à especificidade, entre outras, estes atletas provavelmente possuíam maiores percentuais de fibras musculares do tipo I quando comparados com ciclistas de sprint ${ }^{21}$.

O TAW possui característica predominantemente anaeróbia-glicolítica para a ressíntese de ATP, resultando alta produção de metabólitos como o lactato, que se deve, entre outros, ao aumento das contrações musculares e à estimulação $\beta$-adrenérgica da musculatura em atividade ${ }^{22}$. Assim, quanto maior a PM maior será a [Lac]-7'. Os valores de hiperlactatemia também parecem se associar e influenciar nas [Lac]-LM, podendo inclusive influenciar na determinação da ILM ${ }^{10}$.

Embora tenhamos obtido baixas correlações e não significativas entre as variáveis de aptidão anaeróbia e aeróbia $(p>0,05)$, observa-se uma associação inversa entre estas variáveis, tendendo a sugerir que quanto maiores as IMEEL, ILM e Pmax, menores os valores de PP e PM, ou seja, atletas que se sobressaem em eventos esportivos que exijam ótima aptidão aeróbia provavelmente não se sobressairão em outros de predominância anaeróbia.

Percebe-se que mesmo aplicando o protocolo LM a partir de um TI do tipo rampa, a curva de lactato ("U") foi semelhante aos demais estudos envolvendo o LM com incremento escalonado. A cinética demonstrou predomínio inicial de remoção do lactato, equilíbrio entre remoção e produção, e por fim um predomínio na produção celular e liberação de lactato no sangue (figura 1$)^{2,7}$.

Os resultados demonstraram que não houve necessidade de realizar incrementos por estágios escalonados, os quais proporcionariam estabilidade lactacidêmica como discutido por Tegtbur, Busse e Braumann ${ }^{5}$. Portanto, pode-se dizer que estes resultados são inovadores, podendo novos estudos ser desenvolvidos utilizando-se de protocolos com incrementos em rampa para análise da [Lac].

A ILM obtida pelo TI rampa não diferiu e apresentou boa correlação (tabela 2) e concordância com a IMEEL (figura 3), que é considerada padrão ouro na identificação de intensidade de exercício correspondente a CA (figura 2). Estes resultados também foram reportados por Pardono ${ }^{10}$. Ainda, a aplicação de um TI rampa durante o protocolo LM também parece interessante para minimizar a duração do teste, como verificado por Pardono ${ }^{10} \mathrm{e}$ Smith et al. ${ }^{9}$, embora o objetivo deste último tenha sido outro. Os testes incrementais de curta duração vêm sendo reportados como adequados para analisar variáveis ventilatórias, bem como obter elevados valores de $\mathrm{Pmax}^{23}$, podendo, de acordo com nossos resultados, também ser utilizado para estudar variáveis de cinética lenta, como [Lac].

A ILM e IMEEL estão de acordo com outros estudos envolvendo estes dois protocolos, embora o TI destes estudos tenham sido escalonados ${ }^{2,5,6,24}$. A identificação da ILM em teste de rampa, e a demonstração de igualdade entre ILM rampa e IMEEL são inéditas. No entanto, destaca-se que o padrão de incremento utilizado (1Watt/6seg) resultou em um incremento de 30 Watts a cada 3 min, sendo esta carga incremental comum à 
maioria dos estudos sobre LM escalonado em cicloergômetro ${ }^{7,10,17}$.

Com relação aos valores de ILM, estão acima dos valores obtidos em trabalhos com estudantes de Educação Física ${ }^{7}$ e corredores velocistas ${ }^{11 .}$ Contudo, as ILM identificadas por Smith et al. ${ }^{9}$, Pardono ${ }^{10} \mathrm{e}$ Simões ${ }^{11}$ ocorreram entre $68,0 \%$ e $73,5 \%$ da Pmax, semelhante ao presente estudo $(68,8 \%)$, podendo estes dados serem úteis para a prescrição de treinamento de ciclistas recreacionais, uma vez que foi observada boa correlação entre ILM e a Pmax $(\mathrm{r}=0,90)$, e entre ILM e MEEL $(\mathrm{r}=0,78)$, além do fato das intensidades relacionadas à CA serem boas indicadoras de performance aeróbia tanto em testes de corrida ${ }^{25}$ quanto em ciclismo ${ }^{23}$.

Dantas et al. ${ }^{26}$ verificaram em cicloergômetro que os valores de Pmax obtidos pelo TI do LM foram significativamente inferiores aos valores encontrados durante um TI sem indução de hiperlactatemia prévia, o que sugere uma possível interferência nos valores percentuais calculados no presente estudo. Todavia, estes autores realizaram o TI do LM a partir de um modelo incremental escalonado, diferentemente do presente estudo que adotou um modelo em rampa, que permite a obtenção de maiores valores de $\operatorname{Pmax}^{23}$. Já Simões ${ }^{11}$ não observou diferenças nos valores de Pmax determinados em testes com e sem hiperlactatemia prévia ao TI em esteira ergométrica.

Para prescrição do treinamento, observamos que a FC-LM ocorreu em média a $85,0 \%$ da FC correspondente à intensidade da Pmax (FC-Pmax), sendo que este percentual também está de acordo com valores obtidos por Pardono ${ }^{10}$ e Simões ${ }^{11}$ de aproximadamente $84 \%$, e próximo do percentual encontrado por Smith et al. ${ }^{9}$ de 89,0\%. Contudo, quando comparada percentualmente com a FC máxima obtida pela equação 220-idade observa-se um decréscimo de praticamente 5\% (de 85,0 para $79,8 \%$ ) em relação aos valores calculados quando utilizamos a FC do Pmax (FC-Pmax), assim como também verificado no estudo de Simões ${ }^{11}$, onde a FC-LM passaria de $84 \%$ da FC-Pmáx para um valor médio de $77,3 \%$.

Caputo et al. ${ }^{27}$ não encontraram diferença entre a FC máxima de ciclistas quando estes realizaram testes em corrida e ciclismo, devendo, as fórmulas de FC máxima, serem empregadas com cautela apenas para outros grupos que não ciclistas. Entretanto, para a amostra estudada, a equação talvez possa ser adequada apenas em testes de corrida, pois além de não ser a especificidade dos ciclistas, aumentaria o estresse cardiovascular em decorrência da maior quantidade de massa muscular envolvida ${ }^{28}$.

As correlações apresentadas na tabela 2 não demonstraram significativa interação entre as intensidades anaeróbias e aeróbias que pudessem satisfatoriamente permitir a análise de algum resultado obtido, entretanto, todas as variáveis passíveis de identificação puderam ser obtidas (minimizando visitas e custos adicionais), analisadas e confrontadas com outros estudos. Igualmente, observou-se que os resultados permitiram o emprego de estimativas percentuais para possíveis prescrições e controle de treinamentos baseados na CA, possibilitando a prescrição quando não for possível realizar testes com mensurações de [Lac] ou ergoespirométricos.

De maneira geral, novos estudos deverão ser desenvolvidos utilizando-se protocolos com incrementos rampeados para análise da [Lac] e identificação da ILM, a fim de confirmar os nossos resultados. No entanto, tendo em vista as limitações do estudo, recomenda-se a realização de novos estudos com maior quantidade de voluntários, bem como intensidades referentes ao teste anaeróbio de Wingate específicas para a população estudada.

\section{CONCLUSÃO}

Os resultados do presente estudo demonstraram a positiva aplicação de um padrão de incremento em rampa sob o protocolo LM, permitindo determinar a capacidade aeróbia (ILM) e se mostrando válido em estimar uma intensidade de exercício correspondente ao MEEL.

Ainda, foi possível viabilizar a predição do MEEL a partir dos percentuais de intensidade e frequência cardíaca em relação à Pmax, o que contribuirá na elaboração e controle do treinamento de ciclistas recreacionais.

Por fim, este modelo de LM com indução prévia de hiperlactatemia a partir do TAW, permitiu identificar tanto a aptidão aeróbia quanto anaeróbia em uma única sessão experimental.

\section{REFERÊNCIAS BIBLIOGRÁFICAS}

1. Granier P, Mercier B, Mercier J, Anselme F, Préfaut C. Aerobic and anaerobic contribution to Wingate test performance in sprint and middle-distance runners. Eur J Appl Physiol 1995;70(1):58-65.

2. MacIntosh BR, Esau S, Svedahl K. The lactate minimum test for cycling: estimation of the maximal lactate steady state. Can J Appl Physiol 2002;27(3):232-249.

3. Inbar O, Bar-Or O, Skinner JS. The Wingate Anaerobic Test. Champaign: Human Kinetics; 1996. 
4. Franchini E. Teste anaeróbio de Wingate: Conceitos e aplicação. Revista Mackenzie de Educação Física e Esporte 2002;1(1):11-27.

5. Tegtbur U, Busse MW, Braumann KM. Estimation of an individual equilibrium between lactate production and catabolism during exercise. Med Sci Sports Exerc 1993;25(5):620-627.

6. Simões HG. Comparação entre protocolos de determinação do Limiar Anaeróbio em testes de pista para corredores. [Dissertação de Mestrado - Programa de Pós-Graduação em Educação Física]. São Carlos (SP): Universidade Federal de São Carlos; 1997.

7. Pardono E, Simões HG, Campbell CSG. Efeitos de variações metodológicas sobre a identificação do lactato mínimo. Rev bras Educ Fís Esp 2005;19(1):25-33.

8. Hiyane WC, Simões HG, Campbell, CSG. Velocidade crítica como um método não invasivo para estimar a velocidade de lactato mínimo no ciclismo. Rev Bras Med Esporte 2006;12(6):381-385.

9. Smith MF, Balmer J, Coleman DA, Bird SR, Davison RCR. Method of lactate elevation does not affect the determination of the lactate minimum. Med Sci Sports Exerc 2002;34(11):1744-1749.

10. Pardono E. Efeito de variações metodológicas na identificação do lactato mínimo e sua validade para estimar intensidades de exercício em estado estável. [Dissertação de Mestrado - Programa de Pós-Graduação em Educação Física]. Brasília (DF): Universidade Católica de Brasília; 2005.

11. Simões HG. Respostas metabólicas e hormonais durante os testes de determinação do limiar anaeróbio individual e lactato mínimo. [Tese de Doutorado - Programa de Pós Graduação em Educação Física]. São Carlos (SP): Universidade Federal de São Carlos; 2002.

12. 12. Denadai BS, Gugliemo LGA, Denadai MLDR. Validade do teste de Wingate para a avaliação da performance em corridas de 50 e 200 metros. Motriz 1997;3(2):83-94.

13. Campbell CSG, Simões HS, Denadai BS. Reprodutibilidade do limiar anaeróbio individual (Iat) e lactato mínimo (Lm) determinados em testes de pista. Rev Bras Ativ Fís Saúde 1998;3(3):24-31.

14. Sotero RC, Campbell CSG, Pardono E, Puga GM, Simões HG. Polynomial adjustment as a new technique for determination of lactate minimum velocity with blood sampling reduction. Rev Bras Cineantropom Desempenho Hum 2007;9(4):327-332.

15. Beneke R. Methodological aspects of maximal lactate steady state-implications for performance testing. Eur J Appl Physiol 2003;89(1):95-99.

16. Beneke R, von Duvillard SP. Determination of maximal lactate steady state response in selected sports events. Med Sci Sports Exerc 1996;28(2):241-246.

17. Balikian Junior P, Neiva CM, Denadai BS. Effect of an acute beta-adrenergic blockade on the blood glucose response during lactate minimum test. J Sci Med Sport 2001;4(3):257-265.

18. Tanaka H, Bassett Jr DR, Swensen TC, Sampedro RM. Aerobic and anaerobic power characteristics of com- petitive cyclists in the United States cycling Federation. Int J Sports Med 1993;14(6):334-338.

19. Okano AH, Dodero SR, Coelho CF, Gassi ER, Silva CC, Okano RO, et al. Efeito da aplicação de diferentes cargas sobre o desempenho motor no teste de Wingate. R Bras Ci e Mov 2001;9(4):07-11.

20. Simões HG, Marcon F, Oliveira F, Campbell CSG, Baldisserra V, Costa Rosa LFBP. Resposta da razão testosterona/cortisol durante o treinamento de corredores velocistas e fundistas. Rev bras Educ Fís Esp 2004;18(1):31-46.

21. Korhonen MT, Cristea A, Alen M, Hakkinen K, Sipila S, Mero A, et al. Aging, muscle fiber type, and contractile function in sprint-trained athletes. J Appl Physiol 2006;101(3):906-917.

22. Stainby WN, Brechue WF, O'drobinak DM. Regulation of muscle lactate production. Med Sci Sports Exerc 1991;23(8):907-911.

23. Bentley DJ, McNaughton LR. Comparison of Wpeak $\mathrm{VO}_{2}$ peak and the ventilation threshold from two different incremental exercise tests: Relationship to endurance performance. J Sci Med Sport 2003;6(4):422-435.

24. Bacon L, Kern M. Evaluating a test protocol for predicting maximum lactate steady state. J Sports Med Phys Fitness 1999;39(4):300-308.

25. Simões HG, Denadai BS, Baldissera V, Campbell CSG, Hill DW. Relationships and significance of lactate minimum, critical velocity, heart rate deflection and $3000 \mathrm{~m}$ track-tests for running. J Sports Med Phys Fitness 2005;45(4):441-451.

26. Dantas de Luca R, Rocha R, Burini RC, Greco CC, Denadai BS. The lactate minimum test protocol provides valid measures of cycle ergometer VO2 peak. J Sports Med Phys Fitness 2003;43(3):279-284.

27. Caputo F; Greco CC; Denadai BS. Efeitos do estado e especificidade do treinamento aeróbio na relação $\% \mathrm{VO}_{2}$ max versus \%FCmax durante o ciclismo. Arq Bras Cardiol 2005;84(1):20-23.

28. Basset FA, Boulay MR. Specificity of treadmill and cycle ergometer tests in triathletes, runners and cyclists. Eur J Appl Physiol 2000;81(3):214-221.

\section{Endereço para correspondência}

Emerson Pardono e Herbert Gustavo Simões

Universidade Católica de Brasília

Programa de Mestrado e Doutorado em Educa-

ção Física - Sala G 119

QS07 LT1 EPCT,

72022-900 - Águas Claras, Taguatinga, DF, Brasil

E-mails: epardono@hotmail.com,

hgsimoes@gmail.com 